Louisiana State University

LSU Digital Commons

10-10-2001

\title{
Readout and control of a power-recycled interferometric gravitational-wave antenna
}

\author{
Peter Fritschel \\ Massachusetts Institute of Technology \\ Rolf Bork \\ California Institute of Technology \\ Gabriela González \\ Pennsylvania State University \\ Nergis Mavalvala \\ California Institute of Technology \\ Dale Ouimette \\ California Institute of Technology
}

See next page for additional authors

Follow this and additional works at: https://digitalcommons.Isu.edu/physics_astronomy_pubs

\section{Recommended Citation}

Fritschel, P., Bork, R., González, G., Mavalvala, N., Ouimette, D., Rong, H., Sigg, D., \& Zucker, M. (2001).

Readout and control of a power-recycled interferometric gravitational-wave antenna. Applied Optics, 40 (28), 4988-4998. https://doi.org/10.1364/A0.40.004988

This Article is brought to you for free and open access by the Department of Physics \& Astronomy at LSU Digital Commons. It has been accepted for inclusion in Faculty Publications by an authorized administrator of LSU Digital Commons. For more information, please contact ir@lsu.edu. 


\section{Authors}

Peter Fritschel, Rolf Bork, Gabriela González, Nergis Mavalvala, Dale Ouimette, Haisheng Rong, Daniel Sigg, and Michael Zucker 


\title{
Readout and control of a power-recycled interferometric gravitational-wave antenna
}

\author{
Peter Fritschel, Rolf Bork, Gabriela González, Nergis Mavalvala, Dale Ouimette, \\ Haisheng Rong, Daniel Sigg, and Michael Zucker
}

\begin{abstract}
Interferometric gravitational-wave antennas are based on Michelson interferometers whose sensitivity to small differential length changes has been enhanced by the addition of multiple coupled optical resonators. The use of optical cavities is essential for reaching the required sensitivity but sets challenges for the control system, which must maintain the cavities near resonance. The goal for the strain sensitivity of the Laser Interferometer Gravitational-Wave Observatory (LIGO) is $10^{-21} \mathrm{rms}$, integrated over a $100-\mathrm{Hz}$ bandwidth centered at $150 \mathrm{~Hz}$. We present the major design features of the LIGO length and frequency sensing and control system, which will hold the differential length to within $5 \times 10^{-14} \mathrm{~m}$ of the operating point. We also highlight the restrictions imposed by couplings of noise into the gravitationalwave readout signal and the required immunity against them. (C) 2001 Optical Society of America

OCIS codes: $120.3180,120.2230$.
\end{abstract}

\section{Introduction}

The interferometric gravitational-wave detectors currently under construction by Laser Interferometer Gravitational-Wave Observatory, (LIGO), ${ }^{1}$ VIRGO, ${ }^{2}$ GEO, ${ }^{3}$ and Tokyo Advanced Medium-Scale Antenna (TAMA) ${ }^{4}$ are expected to reach strain sensitivity levels of $\sim 10^{-22} / \sqrt{\mathrm{Hz}}$ at $150 \mathrm{~Hz}$ over baselines of several hundred meters up to several kilometers. ${ }^{5}$ To achieve this sensitivity, all these interferometers implement a Michelson laser interferometer enhanced by multiple coupled optical resonators. ${ }^{6,7}$ The laser light is typically produced by a solid-state laser source, i.e., Nd:YAG, which is locked to a reference cavity for stabilizing its frequency. ${ }^{8-10}$ Before the light is launched into the interferometric detector, it is passed through one or more triangular ring cavities $^{11-13}$ (called mode cleaners), which clean up the beam spatially and suppress frequency and am-

P. Fritschel and M. Zucker are with the Department of Physics and Center for Space Research, Massachusetts Institute of Technology, Cambridge, Massachusetts 02139. R. Bork, N. Mavalvala, and D. Ouimette are with the LIGO Project, California Institute of Technology, Pasadena, California 91125. G. González is with the Department of Physics, The Pennsylvania State University, University Park, Pennsylvania 16802. H. Rong and D. Sigg (sigg_d@ligo.mit.edu) are with the LIGO Hanford Observatory, P.O. Box 1970 S9-02, Richland, Washington 99352.

Received 6 October 2000.

0003-6935/01/284988-11 $\$ 15.00 / 0$

(C) 2001 Optical Society of America plitude noise at higher frequencies. In the case of LIGO (see Fig. 1) the detector uses Fabry-Perot cavities placed in the arms of the Michelson to boost the signal by bouncing the light forth and back multiple times. ${ }^{14}$ An additional partially transmitting mirror is placed in the input path to form the powerrecycling cavity, ${ }^{15}$ which increases the power incident on the beam splitter and, therefore, decreases the shot-noise contribution to the signal-to-noise ratio of the gravitational-wave signal. To isolate the optical elements (test masses) from disturbances introduced by seismic activities of the ground and to allow for free movement of the test masses in the gravitational-wave frequency band, all detectors implement a seismic isolation system ${ }^{16}$ from which the mirrors are suspended by fibers. ${ }^{17}$ This forms a coupled pendulum system with low eigenmode frequencies and high isolation at frequencies above.

Using optical cavities is essential in reaching the ultimate sensitivity goal, but it requires an active electronic feedback system for keeping them on resonance. The control system must keep the roundtrip length of a cavity near an integer multiple of the laser wavelength so that light newly introduced into the cavity interferes constructively with light from previous round-trips. Under these conditions the light inside the cavity builds up, and the cavity is said to be on resonance. ${ }^{18}$ Attaining high-power buildup in the arm cavities also requires that minimal light be allowed to leave the system through the antisymmetric port so that all the light is sent back in the 


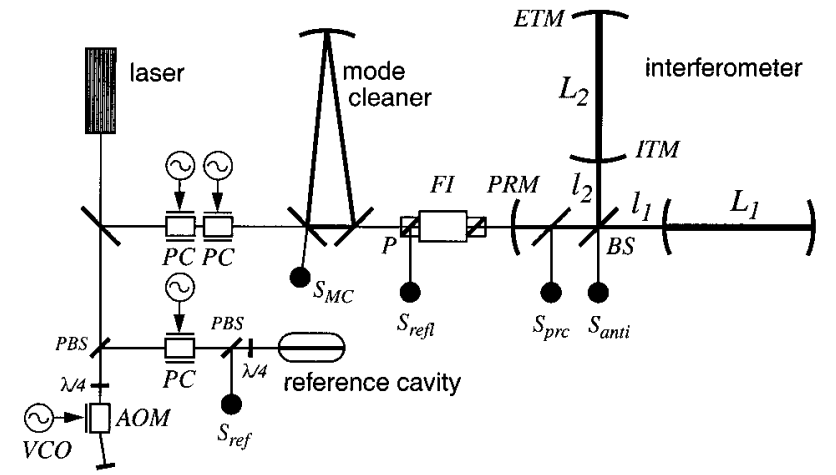

Fig. 1. Schematic view of the optical path in LIGO. The light of a frequency-stabilized Nd:YAG laser is passed through a triangular mode-cleaner cavity before it is launched into a Michelson interferometer. To stabilize the laser frequency, a small fraction of the light is sampled, doubly passed through an AOM that serves as a frequency shifter, passed through a Pockels cell, and sent to a reference cavity. With a polarizing beam splitter (PBS) and quarter-wave plate $(\lambda / 4)$ the light reflected from the reference cavity is measured by a photodetector, which demodulates the signal to obtain the error signal, $S_{\text {ref, }}$ which in turn is used to adjust the laser frequency. The main laser light is passed through a premode cleaner (not shown) and two Pockels cells that impose the phase-modulated rf sidebands used to lock the mode cleaner and the Michelson interferometer. The mode-cleaner locking signal, $S_{\mathrm{MC}}$, is measured by a photodetector in reflection of the mode-cleaner cavity. The light that passes through the mode cleaner is sent through a Faraday isolator (FI) that also serves the purpose-together with a polarizer $(\mathrm{P})$ - to separate out the reflected light signal, $S_{\text {refl }}$. The main interferometer consists of a beam splitter (BS); two arm cavities, each formed by an input test mass (ITM) and an end test mass (ETM); and the power-recycling mirror (PRM). Additional locking signals are obtained at the antisymmetric port, $S_{\text {anti }}$, and by means of sampling a small amount of light from inside the power-recycling cavity, $S_{\mathrm{prc}}$.

direction of the laser where it is reflected back into the system by the power-recycling mirror. Hence an additional feedback loop is needed to control the Michelson phase so that the antisymmetric port is set on a dark fringe.

Implementation of feedback control requires a detection scheme that can separably sense all longitudinal degrees of freedom. Sensing the deviation of a cavity from resonance can be achieved by the PoundDrever-Hall reflection locking technique. ${ }^{8}$ This technique imposes phase-modulated radio-frequency (rf) sidebands on the light incident to a cavity. Typically, only the carrier light is resonant in the cavity, whereas the sidebands are mostly promptly reflected. If the cavity deviates from resonance by a small amount, the carrier light reflected from the cavity acquires an additional phase shift. The cavity then acts as an FM-to-AM converter with the signed amplitude of the AM signal proportional to the length (or frequency) deviation from resonance. The output of a photodetector receiving the light reflected from the cavity is demodulated at the rf frequency to generate an error signal used to feed back to the mirror position or the laser frequency. In LIGO this technique is used to lock various optical cavities: the reference cavity, the pre-mode-cleaner-a fixed-spacer triangular cavity used to filter noise at rf frequencies, the mode cleaner, the cavities in the arms of the Michelson, and the power-recycling cavity (with a variant of the scheme).

Holding the antisymmetric port on a dark fringe is achieved by the Schnupp modulation scheme, ${ }^{19}$ which introduces a macroscopic asymmetry in the path lengths from the beam splitter to the arm cavities. This asymmetry shifts a pair of rf sidebandswhich are made resonant in the power-recycling cavity but not in the arms-away from the dark fringe. Carrier light leaking from the antisymmetric port because of a deviation from a dark fringe will now beat against the always-present rf sidebands, effectively implementing a suppressed carrier scheme. Again, a photodetector demodulating the light at the rf sideband frequency is used to derive the error signal.

In designing the feedback system for a gravitational-wave detector several important points must be considered: (i) the sensitivity of a sensor to a certain degree of freedom; (ii) the sensing noise in the gravitational-wave band; (iii) the noise that is fed back into the detector through the actuation system; (iv) the residual root-mean-square (rms) motion after the feedback system has been engaged; and (v) the largest naturally occurring disturbances that must be corrected by the feedback system. The first two points determine how well a certain degree of freedom can be measured, and in the case of the difference in arm lengths they determine the sensitivity of the instrument to gravitational waves. With the feedback system engaged, sensing noise is added back to the system, and special care must be taken to avoid deteriorating the gravitational-wave sensitivity. This is especially important for auxiliary degrees of freedom that are not directly related to the gravitational-wave signal but may have significant cross couplings. Since noise fluctuations on the input light can couple to the gravitational-wave signal through beating against the light introduced by small offsets from resonance, the feedback system must keep the cavities locked tightly. In the case of the laser amplitude noise and the differential arm-cavity length, the requirement can be as small $10^{-13} \mathrm{~m} .{ }^{20}$ Together with the naturally occurring length and frequency fluctuations this determines how much gain and bandwidth are needed in the feedback paths. At the actuation points the tolerable noise levels together with the largest control signals determine the required dynamic range. Since it is often difficult to build an actuator with high dynamic range and fast response, a hierarchy of actuators might be needed to control a certain degree of freedom. In practice, it may consist of a low-noise high-bandwidth actuator that directly acts on the cavity length in combination with a high-range low-bandwidth outer actuator that keeps the inner one within range. For a scheme like this to work successfully, the larger fluctuations must be concentrated toward lower frequencies. In LIGO one example is given by the tidal actuators, which 
compensate for the deformations of the Earth introduced by the Moon ${ }^{21}$ at the level of $100 \mu \mathrm{m}$, and the mirror position controllers, which keep the arm cavities locked and which have a range of a few micrometers only but have a required sensitivity of $<10^{-19} \mathrm{~m}$ $\sqrt{\mathrm{Hz}}$ at $100 \mathrm{~Hz}$.

There is an ambiguity between the laser wavelength and the length of a cavity; either one can be adjusted to fulfill the resonance condition. However, in a multicavity system adjusting the laser wavelength will simultaneously change the ruler with which all cavities are measured. Furthermore, true frequency noise can couple into the gravitational-wave signal through an imbalance of the arm-cavity reflectivity or storage time. ${ }^{20}$ This leads to the following question: What is the best ruler to use? We will see below that the answer to this question is frequency dependent. This fact will explain the most complex and ambitious feedback system in the LIGO interferometer, the commonmode control loops. This feedback system involves the laser, the mode cleaner, the common-mode motion of the arm cavities, and the power recycling cavity; it implements multiple sensing and actuation points with multiple crossovers in the feedback path, all necessary for the ultimate stabilization of the laser frequency to a level of $3 \times 10^{-7} \mathrm{~Hz} / \sqrt{\mathrm{Hz}}$ at $150 \mathrm{~Hz}$.

\section{Detection Scheme}

The Pound-Drever-Hall reflection locking technique is implemented by means of generating phasemodulated sidebands with a Pockels cell driven by a rf oscillator. If the angular frequency of the rf oscillator is denoted by $\omega_{M}$, the Pockels cell will add a term, $\Gamma \cos \omega_{M} t$, to the phase of the laser light. This leads to symmetric rf sidebands both above and below the laser frequency with amplitudes of $i J_{1}(\Gamma) E_{0} . \quad J_{n}$ denotes the Bessel function of order $n$, and $E_{0}$ is the amplitude of the laser light before it is modulated. The light remaining at the original laser frequency is called the carrier, and its amplitude is $J_{0}(\Gamma) E_{0}$. Since a photodetector measures the power rather than the field strength, it is not able to detect phase modulation on the light. It is, however, sensitive to amplitude modulation, which can be measured by means of down converting (or demodulating) the photocurrent. Multiplying the signal by a cosine function that is derived from the same oscillator that drives the Pockels cell yields-after low-pass filtering-the in-phase term. Similarly, demodulating with a sine function yields the quadrature-phase term.

Locking an optical cavity generally refers to holding the carrier at resonance. The rf sidebands are then typically placed far from resonance. When the carrier is near a resonance, the demodulated output of the reflection photodetector measures a signal that is in amplitude proportional to $\Delta(k L) \simeq \Delta k L+k \Delta L$. In practice, one is often interested in the response of a system as function of angular frequency, $\omega$, where $\omega$ is small compared with the angular frequency of the rf sidebands. If this frequency is also small com- pared with the free spectral range of the cavity, one can use the cavity pole notation to express the frequency dependency of the measured signal. For a high-finesse low-loss cavity that has input and output mirrors with amplitude reflectivity coefficients, $r_{1}$ and $r_{2}$, respectively, the demodulated in-phase signal can be approximated by

$$
\frac{S(\omega)}{S_{0}} \simeq \frac{1-r_{1}^{2}}{\left(1-r_{1} r_{2}\right)^{2}} \frac{1}{1+i \omega / \omega_{\text {pole }}} \Delta(k L),
$$

where the signal was scaled by a factor, $S_{0}=$ $2 J_{0}(\Gamma) J_{1}(\Gamma) P$, that is proportional to the input power, $P$. The angular frequency of the cavity pole, $\omega_{\text {pole }}$, is then given by

$$
\omega_{\text {pole }}=\frac{c}{2 L} \frac{1-r_{1} r_{2}}{\left(r_{1} r_{2}\right)^{1 / 2}} .
$$

The decrease in signal strength at frequencies above the cavity pole is due to the finite width of the resonance. The signal, $S$, has the nice property of linear dependency on the deviation of the cavity from its resonant length (assuming that the laser frequency is fixed and the deviations are small). Hence it can easily be used as an error signal in a control system that feeds back a correction signal to the mirror position.

The sensing scheme of the main interferometer naturally separates common- from differential-mode motions. It is therefore useful to define common and differential length variables and express all error signals as functions of them. If $L_{1}$ is the length of the in-line arm cavity, $L_{2}$ the length of the off-line arm cavity, $l_{1}$ the length between the power-recycling mirror and the input mirror of the in-line arm cavity, and $l_{2}$ the length between the power-recycling mirror and the input mirror of the off-line arm cavity, the common arm length, $L_{+}$, the differential arm length, $L_{-}$, the power-recycling-cavity length, $l_{+}$, and the Michelson length, $l_{-}$, can be expressed as

$$
\begin{aligned}
L_{+}=\frac{L_{1}+L_{2}}{2}, & L_{-}=\frac{L_{1}-L_{2}}{2}, \\
l_{+}=\frac{l_{1}+l_{2}}{2}, & l_{-}=\frac{l_{1}-l_{2}}{2} .
\end{aligned}
$$

We distinguish microscopic from macroscopic lengths-where we assume that the macroscopic lengths exactly fulfill the resonance and dark fringe conditions-by denoting microscopic lengths with a $\Delta$ in front of the corresponding length variable.

The length detection scheme of the LIGO interferometer ${ }^{22-24}$ uses a single set of phasemodulated rf sidebands, whose frequency is chosen to be resonant in the power-recycling cavity but not in the arm cavities. However, the carriers experience a double resonance: They resonate in both the arm cavities and the power-recycling cavity. Three photodetectors are needed to sense all longitudinal degrees of freedom. They detect the beam on the antisymmetric side of the beam splitter (denoted by anti), the beam reflected from the power-recycling 
mirror (denoted by refl), and a small fraction of the light circulating in the power-recycling cavity (denoted by prc). When deriving the sensor signals, we use the following conventions: (i) Only rf signals at the modulation frequency are included in the result; (ii) the modulation is done with a cosine function and, thus, cosine terms in the result are in-phase contributions, whereas sine terms are quadrature-phase contributions; (iii) each signal is given as a complex function of frequency, where the absolute value represents the signal amplitude and its argument represents the phase of the signal relative to the input disturbance; and (iv) the signal at the powerrecycling-cavity port is given without including the factor accounting for the amount of light actually picked off from the main beam. The scope of this paper does not cover angular degrees of freedom ${ }^{25}$ or effects that are introduced by imperfect optics. ${ }^{26}$ For all derivations it was assumed that the losses in the system are negligible and that the optical components are perfectly aligned. For the arm cavities, where the losses cannot be neglected, the amplitude reflectivity coefficients of the end mirrors are lowered to model an effective loss.

It is useful to first define some frequently used quantities. The power-recycling cavity can be related to a simple Fabry-Perot cavity when the Michelson and the two arm cavities are treated as a compound mirror with amplitude reflectivities $r_{c}$ for the carrier and $r_{M}$ for the rf sidebands, respectively. In the case of the carrier the reflectivity is determined by the resonant reflectivity of the arm cavities, whereas for the rf sidebands the reflectivity is determined by the Michelson asymmetry:

$$
r_{c}=\frac{r_{1}-r_{2}}{1-r_{1} r_{2}}, \quad r_{M}=\cos \frac{2 \omega_{M} l_{-}}{c},
$$

where $r_{1}$ and $r_{2}$ are the input and the rear mirror reflectivity of the arm cavities (assumed to be identical for both cavities) and $\omega_{M}$ is the angular frequency of the modulation signal. In principle, the carrier amplitude reflectivity can either be positive (generally referred to as undercoupled), negative (overcoupled), or zero (critically matched); however, the LIGO arm cavities are strongly overcoupled. We also define the quantities, $r_{c}{ }^{\prime}$ and $\hat{r}_{c}$, which are the derivatives of the arm-cavity reflected field with respect to the phase for carrier and rf sidebands, respectively:

$$
r_{c}^{\prime}=\frac{\left(1-r_{1}^{2}\right) r_{2}}{\left(1-r_{1} r_{2}\right)^{2}}, \quad \hat{r}_{c}=\frac{\left(1-r_{1}^{2}\right) r_{2}}{\left(1+r_{1} r_{2}\right)^{2}},
$$

where we assumed that the carrier is exactly resonant and that the rf sidebands are located exactly between resonances. Using $r_{5}$ and $t_{5}$ to denote the amplitude reflectivity and transmission coefficient of the power-recycling mirror, respectively, one can write the amplitude recycling gains, $g_{\text {cr }}$ (carrier) and $g_{\mathrm{sb}}$ (rf sidebands), the amplitude reflectivity coefficients for reflection from the power-recycling mirror, $r_{\mathrm{cr}}$ and $r_{\mathrm{sb}}$, and the amplitude transmission coefficients to the antisymmetric port, $t_{\mathrm{cr}}$ and $t_{\mathrm{sb}}$, as

$$
\begin{aligned}
g_{\mathrm{cr}} & =\frac{t_{5}}{1+r_{5} r_{c}}, & r_{\mathrm{cr}} & =\frac{r_{5}+r_{c}}{1+r_{5} r_{c}}, \\
g_{\mathrm{sb}} & =\frac{t_{5}}{1-r_{5} r_{M}}, & r_{\mathrm{sb}} & =\frac{r_{5}-r_{M}}{1-r_{5} r_{M}}, \\
t_{\mathrm{cr}} & =0, & t_{\mathrm{sb}} & =\frac{t_{5} \sqrt{1-r_{M}^{2}}}{1-r_{5} r_{M}} .
\end{aligned}
$$

The following notation is used to account for the armcavity pole, $\omega_{c} \sim 2 \pi \times 100 \mathrm{~Hz}$, and for the pole of the double resonance, $\omega_{c c} \sim 2 \pi \mathrm{Hz}$,

$$
\begin{aligned}
s_{c}=i \frac{\omega}{\omega_{c}}, & \omega_{c}=\frac{c}{2 L_{+}} \frac{1-r_{1} r_{2}}{\sqrt{r_{1} r_{2}}}, \\
s_{c c}=i \frac{\omega}{\omega_{c c}}, & \omega_{c c}=\frac{1+r_{5} r_{c}}{1+r_{5}} \omega_{c} .
\end{aligned}
$$

The signal at the antisymmetric port is sensitive only to differential arm length and Michelson length changes. It can be expressed as

$$
\begin{aligned}
\frac{S_{\mathrm{anti}}}{S_{0}}= & -4 g_{\mathrm{cr}} t_{\mathrm{sb}} r_{c}^{\prime} k \Delta L_{-} \frac{1}{1+s_{c}} \sin \omega_{M} t \\
& +4 g_{\mathrm{cr}} t_{\mathrm{sb}} r_{c} k \Delta l_{-} \frac{1}{1+s_{c}} \sin \omega_{M} t .
\end{aligned}
$$

Both components of this signal are in the quadrature phase and are filtered by the arm-cavity pole. For an arm-cavity-length change this is obvious, since the signal that is produced by the arm cavities falls outside the cavity linewidth at higher frequencies and, thus, its buildup is reduced. For the Michelson length, the signal is produced in the power-recycling cavity and the attenuation comes from the additional phase shift that the light experiences upon reflection from the arm cavities for frequencies away from resonance. This then transforms the AM signal at the antisymmetric port into FM, thus effectively reducing the measured signal strength.

At the reflection port the in-phase signal is sensitive to changes in the common arm-cavity length and the power-recycling-cavity length, whereas the quadrature-phase signal is sensitive mostly to the Michelson length. It can be written as

$$
\begin{aligned}
\frac{S_{\text {refl }}}{S_{0}}= & -4 g_{\mathrm{sb}} t_{\mathrm{sb}} r_{\mathrm{cr}} \hat{r}_{c} k \Delta L_{-} \sin \omega_{M} t \\
& -4 g_{\mathrm{sb}} t_{\mathrm{sb}} r_{\mathrm{cr}} k \Delta l_{-} \sin \omega_{M} t \\
& +4 g_{\mathrm{cr}}{ }^{2} r_{\mathrm{sb}} r_{c}{ }^{\prime} k \Delta L_{+} \frac{1}{1+s_{c c}} \cos \omega_{M} t-4\left(g_{\mathrm{cr}}{ }^{2} r_{\mathrm{sb}} r_{c}\right. \\
& \left.+g_{\mathrm{sb}}{ }^{2} r_{\mathrm{cr}} r_{M}\right) k \Delta l_{+} \frac{1+s_{r}}{1+s_{c c}} \cos \omega_{M} t,
\end{aligned}
$$


where the zero in the transfer function of the powerrecycling-cavity length is given by

$$
s_{r}=i \frac{\omega}{\omega_{r}}, \quad \omega_{r}=\left(1+\frac{g_{\mathrm{cr}}^{2} r_{\mathrm{sb}} r_{c}}{g_{\mathrm{sb}}^{2} r_{\mathrm{cr}} r_{M}}\right) \omega_{c c} .
$$

One can see from the above equations that common and differential degrees of freedom are clearly separated-downconverting the signal will project out either the in-phase part (demodulating with a cosine) or the quadrature-phase part (demodulating with a sine). Since the differential signals in the quadrature-phase are produced by a change in the rf sidebands, which are then beating against the static carrier field, there is no frequency dependency of these signals below the pole of the power-recycling cavity, and their signs depend only on the coupling of the carrier field into the interferometer. In particular, if the carrier is critically matched, the quadrature-phase signals become identically zero.

Because the in-phase signal originating from a change of common arm-cavity length is caused by a change of the carrier field alone, it is affected by the double cavity pole only. The situation is more complicated for the in-phase signal originating from a power-recycling-cavity length. Here, both the carrier and the rf sidebands contribute to the signal, however with an important difference: The carrier experiences the double resonance, whereas the $\mathrm{rf}$ sidebands do not. Adding the two signals together gives a transfer function consisting of a term accounting for the double cavity pole and an additional term with a zero at frequency $\omega_{r}$. As can be seen from Eq. (11), the frequency of the zero can be positive or negative. In control system theory the case of negative frequency is generally referred to as a non-minimumphase system ${ }^{27}$; it tends to make the feedback system unstable - or at least difficult to control-because it causes the signal amplitude to rise toward higher frequencies while simultaneously introducing a phase lag. However, we will see below that it can be canceled out by an appropriate choice of feedback topology and by establishment of a gain hierarchy. It should be noted that the signs of the in-phase signals do not depend on the coupling of the carrier into the interferometer but rather on the coupling of the rf sidebands into the power-recycling cavity (common arm-cavity length) and the ratio of the powerrecycling gains of carrier and rf sidebands (powerrecycling-cavity length), respectively.

At the power-recycling-cavity port the situation is similar to the one in reflection; the signal reads

$$
\begin{aligned}
\frac{S_{\mathrm{prc}}}{S_{0}}= & +4 \frac{g_{\mathrm{cr}} g_{\mathrm{sb}}}{t_{5}} t_{\mathrm{sb}} \hat{r}_{c} k \Delta L_{-} \sin \omega_{M} t \\
& +4 \frac{g_{\mathrm{cr}} g_{\mathrm{sb}}}{t_{5}} t_{\mathrm{sb}} k \Delta l_{-} \sin \omega_{M} t
\end{aligned}
$$

$$
\begin{aligned}
& -4 \frac{g_{\mathrm{cr}}^{2} g_{\mathrm{sb}}}{t_{5}} r_{M} r_{c}{ }^{\prime} k \Delta L_{+} \frac{1}{1+s_{c c}} \cos \omega_{M} t \\
& +4 \frac{g_{\mathrm{cr}} g_{\mathrm{sb}}}{t_{5}}\left(g_{\mathrm{cr}}-g_{\mathrm{sb}}\right) k \Delta l_{+} \frac{1+s_{p}}{1+s_{c c}} \cos \omega_{M} t,
\end{aligned}
$$

where the zero in the transfer function of the powerrecycling-cavity length is given by

$$
s_{p}=i \frac{\omega}{\omega_{p}}, \quad \omega_{p}=\left(1-\frac{g_{\mathrm{cr}}}{g_{\mathrm{sb}}}\right) \omega_{c c} .
$$

There are some important differences, however. The quadrature-phase signal from the Michelson length does not depend on the coupling of the carrier into the interferometer anymore, and it is nonzero even when the carrier is critically coupled. The sign of the signal from the power-recycling-cavity length depends only on the relative size of the powerrecycling gain of carrier and rf sidebands. The same is true for the location of the zero: If the carrier power-recycling gain is higher than that of the $\mathrm{rf}$ sidebands, the frequency of the zero is positive.

One problem with this scheme is immediately obvious when we look at the relative strength of the common-mode signals. Both in reflection and at the power-recycling-cavity port the signal that is due to common arm-cavity-length changes dominates over the signal from power-recycling-cavity length. In LIGO the problem is solved by use of the signal in reflection to feed back into the laser frequency with a high-bandwidth $(\sim 20-\mathrm{kHz})$ control loop. At frequencies for which the gain of the loop is high the remaining signal at the power-recycling-cavity port is then sensitive to the power-recycling-cavity length alone. If we neglect terms from the differential degrees of freedom and if we set $S_{\text {refl }}=0$, one can solve for $\Delta L_{+}$and substitute it back into Eq. (12),

$$
\begin{aligned}
\left.\frac{S_{\mathrm{prc}}}{S_{0}}\right|_{S_{\mathrm{refl}} \rightarrow 0}= & -4 \frac{g_{\mathrm{sb}}{ }^{2} r_{M}}{t_{5} r_{\mathrm{sb}}}\left(g_{\mathrm{cr}} r_{\mathrm{sb}} r_{c}+g_{\mathrm{sb}} r_{\mathrm{cr}} r_{M}\right) \\
& \times k \Delta l_{+} \cos \omega_{M} t .
\end{aligned}
$$

As an additional benefit the signal has now become frequency independent! Expanding the sum term of Eq. (14) with the help of Eq. (6), one finds

$$
g_{\mathrm{cr}} r_{\mathrm{sb}} r_{c}+g_{\mathrm{sb}} r_{\mathrm{cr}} r_{M} \propto r_{M}+r_{c} .
$$

The sign of the signal depends only on the sum of the arm-cavity amplitude reflection coefficients of carrier and $\mathrm{rf}$ sidebands ( $r_{c}$ is generally made negative). An error signal for the power-recycling-cavity length can be derived as long as the values for these reflectivity coefficients are different from each other.

\section{Noise Couplings}

Requirements related to noise-coupling mechanisms are made by examination of the impact on the LIGO sensitivity goal. Each distinct mechanism is not allowed to degrade this sensitivity curve by more than 
$0.5 \%$, over any frequency range; this means that the equivalent strain-noise amplitude of a technical noise coupling must at all frequencies lie at least a factor of 10 below this curve.

Sensing noise-or more precisely the signal-tonoise ratio of the sensor-limits the amount of suppression that can be achieved by a control system. Shot noise of the light at the antisymmetric port limits the gravitational-wave sensitivity above $\sim 150 \mathrm{~Hz}$ in LIGO. ${ }^{1}$ Technical noise sources such as electronic noise and photodetector nonlinearity or nonuniformity must be kept well below the more fundamental shot-noise level. To maintain maximum optical power in the system - and thus maximum signal-toshot-noise ratio-the control system must hold the cavity lengths closely to their resonant points. In LIGO this sets the requirement for the allowed residual deviations from resonance for the common arm-cavity length, $<2 \times 10^{-12} \mathrm{~m} \mathrm{rms}$, and for the power-recycling-cavity length, $<1 \times 10^{-10} \mathrm{~m} \mathrm{rms}$.

Control actuator noise must also be kept from degrading the overall performance. This puts significant constraints on actuator dynamic range, as will be seen in following sections.

Suppressing laser-frequency noise is the most important feature of the common-mode feedback design. The required level of stability is established by calculation of how frequency noise propagates to the gravitational-wave signal measured at the antisymmetric port. Writing the laser frequency, $f$, as

$$
f=f_{0}+\delta f \cos \omega t,
$$

which includes a noise term of strength $\delta f$ at angular frequency $\omega$, one can write the signal at the antisymmetric port, $S_{\text {anti }}{ }^{\delta}$, owing to laser-frequency noise ${ }^{20}$ as

$$
\begin{aligned}
\frac{S_{\mathrm{anti}}^{\delta f}}{S_{0}}= & -g_{\mathrm{cr}} t_{\mathrm{sb}} \frac{2 \pi \delta f}{\omega}\left\{\left[4 i r_{c} \frac{\omega_{c} l_{-}}{c} \frac{\left(1+s_{c} / r_{c}\right) s_{c}}{1+s_{c c}}\right.\right. \\
& \left.+i \frac{\delta \omega_{c}}{\omega_{c}} \frac{\left(1-r_{c}\right) s_{c}}{\left(1+s_{c c}\right)\left(1+s_{c}\right)}+i \delta r_{c} \frac{s_{c c}}{1+s_{c c}}\right] \\
& \left.\times \sin \omega_{M} t+i r_{\mathrm{sb}} s_{m} \delta r_{c} \cos \omega_{M} t\right\},
\end{aligned}
$$

with $s_{m}=i \omega / \omega_{M} ; \delta \omega_{c}$ and $\delta r_{c}$ are the differences between the arm cavities for cavity pole frequency and amplitude reflectivity, respectively.

Only the quadrature-phase component is important for the gravitational-wave readout. It has three contributions: (i) a term due to the Schnupp asymmetry length, (ii) a term due to the storage-time difference of the arm cavities, and (iii) a term due to the arm-cavity reflectivity difference. The first term is produced by audio-frequency sidebands on the carrier that leak out the antisymmetric port. This signal is filtered by the double cavity resonance up to the arm-cavity pole, above which the audio sidebands are promptly reflected by the arms. The second term is caused by a difference in phase shift acquired by the carrier audio sidebands reflected from the arm cavi- ties when the cavity storage times are not perfectly matched. This signal is filtered by both the double cavity resonance, which affects the size of the carrier audio sidebands in the recycling cavity, and the armcavity pole, which affects their coupling into the arm cavities. The third effect has a different origin: The difference in reflectivity between the arm cavities causes carrier light to leak out the antisymmetric port as a contrast defect. In the absence of power recycling, this would not create a signal, because the audio sidebands of the carrier and rf sidebands cancel each other. However, above the double cavity pole the audio sidebands of the carrier are filtered away, leaving only the rf audio sidebands, now beating with the static carrier contrast defect to produce a signal. In practice, the third term dominates the error budget and is solely responsible for the requirement of frequency-noise suppression. Assuming a difference in reflectivity of $0.5 \%$ the laser frequency must be stabilized to $3 \times 10^{-7} \mathrm{~Hz} / \sqrt{\mathrm{Hz}}$ or lower at $150 \mathrm{~Hz}$.

In principle, either the signal in reflection or the signal at the power-recycling-cavity port could be used to stabilize the laser frequency. However, the shot-noise contribution at the power-recycling-cavity port is too large and would limit the frequency noise to a level not compatible with the requirement. The shot-noise contribution to the light in reflection is smaller because the power-recycling mirror is chosen so that most of the light is used up inside the interferometer rather than sent back to the laser. Furthermore, one would like to keep the loss introduced by sampling the power-recycling cavity-and thus its signal strength-as low as possible to avoid degrading the gravitational-wave sensitivity. The signal in reflection, $S_{\text {refl }}$, due to frequency noise then reads as

$$
\frac{S_{\mathrm{reff}}{ }^{\delta f}}{S_{0}}=2 r_{\mathrm{sb}}\left(1-r_{\mathrm{cr}}\right) \frac{2 \pi \delta f}{\omega_{c c}} \frac{1}{1+s_{c c}} \cos \omega_{M} t .
$$

Amplitude noise on the incident laser light can couple to the gravitational-wave readout signal through a differential length offset in the arm cavities. When one makes the Ansatz, $E=E_{0}(1+\Delta A / A) \cos \omega t$, for the laser amplitude noise, the quadrature-phase signal, $S_{\text {anti }}{ }^{A}$, at the antisymmetric port becomes

$$
\begin{aligned}
\frac{S_{\mathrm{anti}}{ }^{A}}{S_{0}}= & -4 g_{\mathrm{cr}} t_{\mathrm{sb}} r_{c}{ }^{\prime} \frac{\Delta A}{A} k \Delta L_{-} \\
& \times\left[1+\frac{1}{\left(1+s_{c c}\right)\left(1+s_{c}\right)}\right] \sin \omega_{M} t .
\end{aligned}
$$

Setting requirements for adequately controlling this noise coupling involves a trade-off between stabilizing the laser power and suppressing differentialmode motions of the interferometer. In LIGO the trade-off is made by setting the maximum allowed deviations from resonance for the differential armcavity length to $5 \times 10^{-14} \mathrm{~m} \mathrm{rms}$ and requiring the relative laser power fluctuations to be smaller than $8 \times 10^{-8} \mathrm{~Hz}^{-1 / 2}$ above $150 \mathrm{~Hz}$. A similar argument holds for the relative amplitude noise of the rf master 
oscillator used to generate the rf sidebands. Its requirement is that the amplitude fluctuations be below $4 \times 10^{-8} \mathrm{~Hz}^{-1 / 2}$ above $150 \mathrm{~Hz}$. Finally, the requirement on the phase noise of the rf master oscillator is determined by the conversion of this phase noise into amplitude noise in passing through the mode cleaner, assuming that the rf frequency is not an exact integer multiple of the free spectral range. With an absolute frequency offset of the rf master oscillator no larger than $100 \mathrm{~Hz}$, the oscillator phase noise must be smaller than $6 \times 10^{-5} \mathrm{rad} / \sqrt{\mathrm{Hz}}$ at 150 $\mathrm{Hz}$.

\section{Environmental Influences}

Seismic motion of the ground is many orders of magnitude larger than the required gravitational-wave sensitivity in the frequency band of interest. ${ }^{28} \mathrm{In}$ LIGO a multistage passive seismic isolation stack $^{16}$ together with a single-stage pendulum suspension system $^{17}$ is used to isolate the optical components from ground vibrations. This system works well for frequencies above $\sim 1 \mathrm{~Hz}$ but gives no suppression at frequencies below.

The bulk of microseismic motion, between 0.3 and $3 \mu \mathrm{m}$ in rms amplitude, occurs at frequencies below 1 $\mathrm{Hz}$ and peaks prominently at $\sim 0.15 \mathrm{~Hz}$. This largely determines the required dynamic range of actuation systems to control the mirror positions. One of the actuators acts directly on the mirror: Four permanent magnets are bonded to the back of each suspended mirror, and corresponding coils, mounted on the isolation stack, are used to control the mirror position by application of an electromagnetic force. Since these actuators must have low noise in the gravitational-wave band, their range is limited to $\sim 10 \mu \mathrm{m}$.

The motions of the ground due to tidal deformations of the Earth by the Moon can be as large as $\sim 0.5$ $\mathrm{mm}$ over a baseline of $4 \mathrm{~km} .{ }^{21}$ But, because of the long period, a different actuation system can be used. For tidal components that change the average length of the two arms of the interferometer it is always possible to adjust the laser frequency (by an amount of order $30 \mathrm{MHz}$ ). Differential tidal components are tracked by piezoelectric transducers (that are able to move the whole seismic isolation system relative to the ground. The bandwidth of these piezoelectric transducer actuators is $\sim 10 \mathrm{~Hz}$, which makes them also useful for reducing motions at microseismic frequencies.

Mechanical resonances in the seismic isolation and mirror suspension systems can significantly increase the optic motion in a narrow band. This is particularly true for the first stack resonance at $\sim 1.2 \mathrm{~Hz}$ and the vertical bounce mode of the suspensions at $\sim 13$ $\mathrm{Hz}$. For the latter, the curvature of the Earth's surface along a 4-km baseline gives rise to a $\sim 3 \times 10^{-4}$ rad vertical-to-horizontal coupling coefficient. Another such coupling can be introduced by vertically oriented wedge angles of the mirror surfaces. These wedges are necessary to deflect ghost beams, which are produced by the minimal-reflecting backsurfaces of the mirrors away from the main beam. LIGO uses vertical wedges with typical angles around $\sim 20$ mrad, which add an inclination to the beam in the power-recycling cavity, which in turn gives rise to a coupling coefficient of $\sim 2 \times 10^{-2} \mathrm{rad}$. Since both the stack resonances and the vertical bounce modes are within the bandwidth of the control system, their suppression can be enhanced by means of implementing resonant gain stages in the compensation path.

Another set of resonances to be considered are the violin modes of the suspension wires and the internal modes of the optical components. These resonances are typically out of band of the control system. In the best case (violin modes) they add just a little additional motion, but in the worst case (internal modes) they can make the control system unstable. Even a small coupling from the mirror feedback signal to one of these high- $Q$ modes can bring the gain in the control system above unity at the mode frequency. If this happens above the control system bandwidth, it can lead to an unbound oscillation that must be "notched" out by the electronics of the control system.

The limit for position fluctuations in suspended optics is given by thermal noise. ${ }^{17,29}$ In LIGO the thermal noise of the suspensions limits the gravitational-wave sensitivity in the frequency band between 40 and $150 \mathrm{~Hz}$. Because the laser frequency is stabilized by locking of the laser first to the reference cavity, then to the mode cleaner, and finally to the common arm-cavity length, thermal noise can limit the accuracy of these cavities as frequency standards. For LIGO the thermal-noise-limited frequency stability at $100 \mathrm{~Hz}$ is $\sim 10^{-4} \mathrm{~Hz} / \sqrt{\mathrm{Hz}}$ for the mode cleaner and $\sim 10^{-8} \mathrm{~Hz} / \sqrt{\mathrm{Hz}}$ for the common mode of the interferometer.

\section{Feedback Compensation Network}

The required level of frequency stability is achieved through three cascaded stabilization levels: first, prestabilization to a short rigid reference cavity; second, stabilization to a much longer, suspended-mirror mode-cleaner cavity; third, stabilization to the long interferometer arms cavities. At each stage, frequency fluctuations are reduced by a factor of $\sim 1000$ at $100 \mathrm{~Hz}$. The combined multiplicative suppression brings the raw laser noise of $10^{2} \mathrm{~Hz} / \sqrt{\mathrm{Hz}}$ at 100 $\mathrm{Hz}$ down to the required level. The cascaded stabilization approach is also critical to achieving frequency stability at high frequencies, since at $\sim 10$ $\mathrm{kHz}$ a total suppression of $100 \mathrm{~dB}$ is required.

The suspended mode-cleaner cavity is a good frequency standard in the gravitational-wave band where its ultimate performance is limited by either suspension thermal noise or by shot noise in the photodetector. But at lower frequencies, seismic excitations dominate the movement of the mirrors and the rigid reference cavity makes a better standard. Hence the mode-cleaner control system electronics (see Fig. 2) is split into two paths that cross over at a few hertz: a low-frequency path that adjusts the mode-cleaner length to the laser frequency and a 


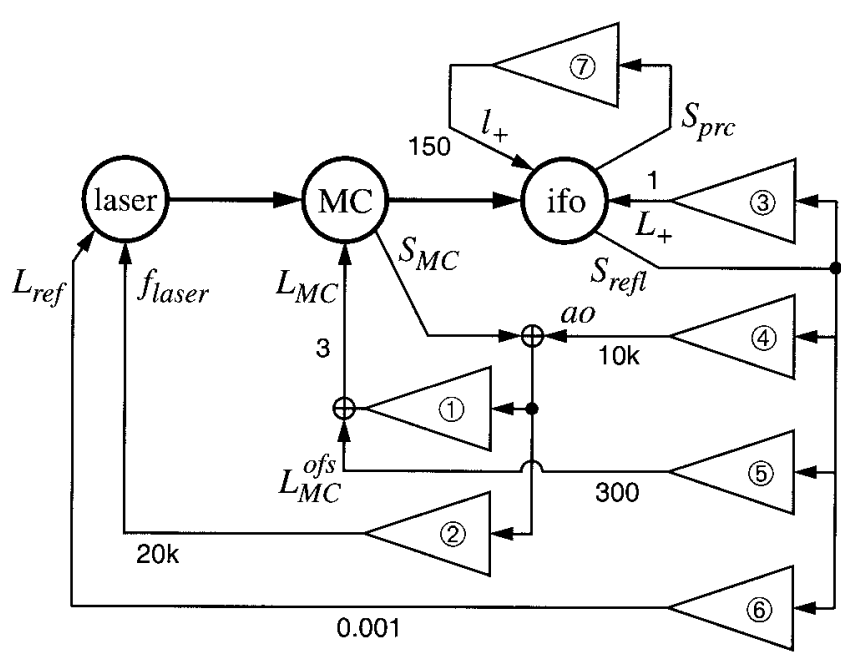

Fig. 2. Common-mode control system. The mode-cleaner error signal, $S_{\mathrm{MC}}$, is split into two paths: the mode-cleaner length path (1) feeding back to the position of a mode-cleaner mirror, $L_{\mathrm{MC}}$, and the laser path (2) feeding back to the laser frequency, $f_{\text {laser }}$, with the VCO-AOM. The in-phase reflection signal, $S_{\text {refl }}$, of the interferometer (ifo) is split into four paths: the arm-cavity path (3) feeding back to the common arm-cavity mirror positions, $L_{+}$; the additive offset (ao) path (4) feeding back to the error point of the mode-cleaner control system; the mode-cleaner length offset path (5) feeding back to the mode-cleaner mirror position, $L_{\mathrm{MC}}{ }^{\text {ofs }}$; and the tidal path (6) feeding back to the reference cavity length, $L_{\text {ref }}$, with the thermal actuator. The in-phase signal at the powerrecycling-cavity port, $S_{\text {prc }}$, is mostly sensitive to the powerrecycling-cavity length, $l_{+}$, and is fed back to the recycling mirror position (7). The numbers in the feedback paths indicate unity gain frequencies in hertz.

high-frequency path that adjusts the laser wavelength to the mode-cleaner length. The laser wavelength is adjusted through an acousto-optical modulator (AOM), which serves as a variable frequency shifter. The AOM is driven by a voltagecontrolled oscillator (VCO), and the combination can shift the laser frequency over a $20-\mathrm{MHz}$ range. Only the small fraction of the main beam that is used for the frequency prestabilization passes through the AOM. Changing the frequency of the AOM will change the wavelength of the light incident on the reference cavity; since the reference cavity error signal is fed back into the laser with a high-bandwidth feedback loop, the laser frequency tracks the frequency shift introduced by the AOM, effectively changing the wavelength of the light sent to the mode cleaner.

The split feedback arrangement is essential to limit fluctuations of the mode-cleaner output light below a few hertz, but it does come at a price-the path that controls the mode-cleaner length does not serve to stabilize the frequency, and even when this path is not dominant it can limit the frequency suppression. If $G_{1}$ is the open-loop gain of the mode-cleaner length path, and $G_{\mathrm{mc}}$ is the open-loop gain of the complete mode-cleaner control system, then the frequencysuppression factor provided by this feedback loop is given by $\left(1+G_{\mathrm{mc}}\right) /\left(1+G_{1}\right)$. Clearly the gain $G_{1}$ must be rolled off quickly above the cross-over frequency in order to realize the full benefit of a high loop gain $G_{\mathrm{mc}}$.

The best frequency standard in the gravitationalwave band is the average length of the arm cavities. Again, this frequency standard is not a good standard at low frequencies (where seismically excited motion is large), and the controller is split into a path feeding back into the arm-cavity mirror position at frequencies below $\sim 1 \mathrm{~Hz}$ and a path feeding back to the laser. The feedback path to the laser is complicated by the fact that both the laser wavelength and the modecleaner length must follow the common arm-cavity length. Rather than feeding back directly to the laser, the controller is split again into a path acting on the mode-cleaner length and an additive offset path adding correction signals to the mode-cleaner error point. Both of these paths are inherently ac coupled, on account of the action of the mode-cleaner control system's own length path $\left(G_{1}\right)$. The resulting changes in the mode-cleaner length in turn produce a signal that is fed back to the laser wavelength. The bandwidth of the mechanical path is limited by the internal resonances of the mode-cleaner mirror that is actuated. The error point offset path serves to extend the bandwidth of the common-mode control system; this actuator has a very small range-it can pull the frequency only a small fraction of the modecleaner linewidth - and so cannot be used to correct large low-frequency fluctuations, but its bandwidth is limited only by the bandwidth of the mode-cleaner controller feeding back to the laser. The cross over is made at a few hundred hertz, above which the frequency fluctuations are instrinsically small. The bandwidth of the additive offset path is $10 \mathrm{kHz}$, and the frequency suppression at $100 \mathrm{~Hz}$ is $\sim 70 \mathrm{~dB}$.

A plot of the modeled residual frequency noise of the LIGO common-mode feedback system and its largest contributors is shown in Fig. 3 . The dominant contribution above $\sim 10 \mathrm{~Hz}$ is due to the lack of suppression of the incoming frequency noise from the mode cleaner, with some added components from shot noise. It is however below the level that would degrade the gravitational-wave sensitivity to expected sources.

Feeding back to the arm-cavity mirrors becomes unfeasible at tidal frequencies, because the suspension controllers run out of range. Increasing the range is not practical, because it would sacrifice performance at the gravitational-wave frequencies. Therefore the common-mode feedback of the arm cavities includes yet another path that at frequencies below $1 \mathrm{mHz}$ is fed back again to the laser frequency. Here again, though, the range of the VCO-limited by need to maintain low phase noise in the oscillator-is not sufficient. Instead the temperature of the rigid reference cavity is changed to track the commonmode tidal distortions; thermal expansion then changes the reference cavity length, which forces the frequency stabilization controller to drag the laser frequency along with it.

In contrast to the laser-frequency controls, the 


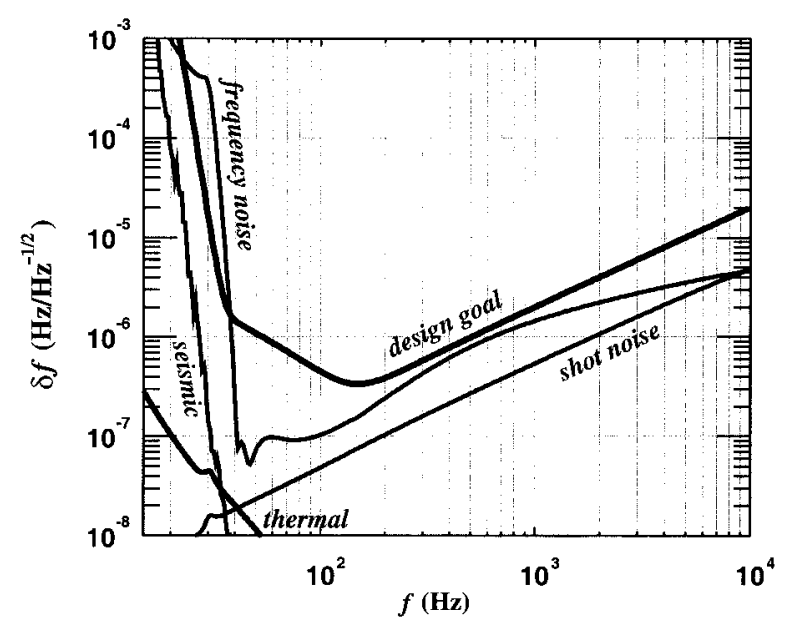

Fig. 3. Residual frequency noise. Shown is the residual frequency noise of the light incident on the interferometer with contributions from the laser-frequency noise, the seismic motions, the suspension thermal noise, and the photon shot noise at the reflection port. The design goal corresponds to one tenth of the design strain sensitivity at the antisymmetric port. With the exception of a small region between 20 and $40 \mathrm{~Hz}$ the sum of all contributions is below the design requirement. The frequency noise is given as a spectral density with units of hertz per root hertz.

feedback system of the power-recycling-cavity length is relatively simple. The error signal derived from the power-recycling-cavity port is fed back to the position of the recycling mirror with a bandwidth of $\sim 150 \mathrm{~Hz}$ and a gain at dc of $\sim 120 \mathrm{~dB}$.

The differential-mode feedback-compensation network is shown in Fig. 4. The signal at the antisymmetric port is fed back to the differential arm-cavity length with a bandwidth of $\sim 300 \mathrm{~Hz}$ and a gain at dc of $\sim 200 \mathrm{~dB}$. This channel naturally contains the

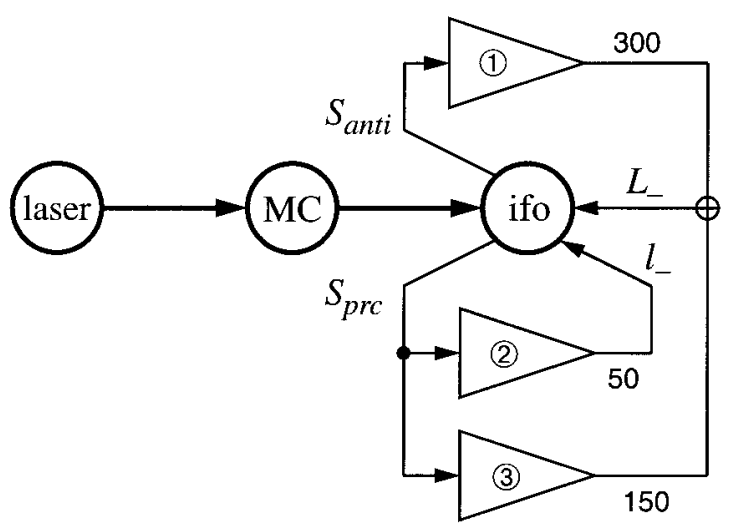

Fig. 4. Differential-mode control system. The quadrature-phase signal at the antisymmetric port, $S_{\text {anti }}$, is used to feed back to the differential arm-cavity length, $L_{-}$. The $L_{-}$feedback control signal is split into a path to the mirror positions of the arm cavities and into a low-frequency path to the PZT tidal actuators (not shown). The quadrature-phase signal at the power-recyclingcavity port, $S_{\text {prc }}$, is split into two paths: the Michelson path (2) fed back to the beam-splitter position, $l_{-}$, and an off-diagonal compensation path (3) fed back to differential arm-cavity length. The numbers in the feedback paths indicate unity gain frequencies in hertz. gravitational-wave signal, and it is important to consider where in the signal chain the signal is read out. In principle either the error or control signal can be used, in each case correcting for the frequencydependent loop gain to recover the original disturbance signal. For the control signal the correction would be small (and relatively independent of the feedback gain) at low frequencies where the loop gain is high; for the error signal the situation is reversedthe correction is small at high frequencies, where the loop gain is small. Given that the error and control signals are connected by a known, stable transfer function, in general either signal can be used for the readout without penalty. A potential discriminant exists if there is significant electronic noise injected between the error and control signals; its effect is reduced by the loop gain in the control-signal monitor but not in the error-signal monitor.

The signal sampling the light of the powerrecycling cavity is produced by a reflection from the second (wedged) surface of an input test mass. Since any type of loss in the power-recycling cavity reduces the effectiveness of the recycling scheme, only $\sim 300$ parts in $10^{6}$ of the light is actually deflected off to form the power-recycling-cavity signal. This has the effect that the shot-noise contribution to this signal is relatively large. Since the powerrecycling-cavity signal is used to feed back to the beam-splitter position and since the signal at the antisymmetric port has a small but nonnegligible sensitivity to the Michelson length, the noise propagating from the power-recycling-cavity port to the beam-splitter position can degrade the gravitationalwave sensitivity. To avoid such a performance deterioration, a small fraction of the power-recyclingcavity signal is also fed back to the differential armcavity length so that the Michelson control signal becomes truly orthogonal to the gravitational-wave readout. The bandwidth of this control system is around $50 \mathrm{~Hz}$, and the gain at dc is $\sim 110 \mathrm{~dB}$. Figure 5 shows the LIGO design sensitivity and noise contributions from laser-frequency noise and shot noise introduced through the power-recycling-cavity port. It was assumed that the off-diagonal compensation path suppresses the latter contribution by a factor of 10. In reality, one might actually do better, which will bring this curve down even further.

The $L_{-}, l_{-}, L_{+}$, and $l_{+}$control paths shown in Figs. 2 and 4 hide an additional feature of the controls scheme. These degrees of freedom are derived from the positions of six optics, but the interferometer generates only four length error signals, so the controls problem is underconstrained. The position of each suspended optic with respect to its support structure is measured with optical sensors. These signals can be used to actively damp the axial pendulum mode (as well as the transverse and angular modes), with the same magnet-coil actuators used for the interferometer control. Given that four optic positions can be controlled with the interferometer error signals, two of the optics are then controlled with this local damping system. Since the signal-to-noise ra- 


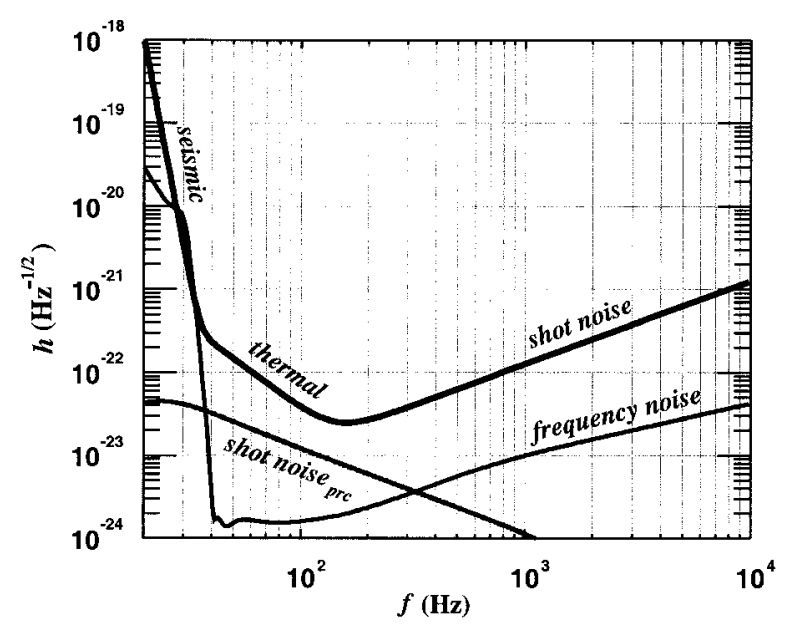

Fig. 5. LIGO sensitivity curve. The design strain sensitivity is plotted against frequency outlining the three main contributions from seismic motions, suspension thermal noise, and photon shot noise. Also shown are contributions from the laser-frequency noise and from shot noise at the power-recycling-cavity port assuming that the off-diagonal compensation path gives a suppression factor of 10 . The laser amplitude noise will appear at a level of $2 \times 10^{-24} \mathrm{~Hz}^{-1 / 2}$ assuming that the rms of the differential arm-cavity motion is $2 \times 10^{-14} \mathrm{~m}$. The strain is given as an amplitude spectral density with a unit of relative length change per root hertz.

tio of the local sensors is relatively low and some fraction of these sensor signals feed through to the optic when it is under local control, the two optics that couple most weakly to the gravitational-wave signal are locally damped: the beam splitter and the power-recycling mirror. This approach also ensures that there are no large relative motions between the interferometer and the laser source, which could produce spurious signal through upconversion processes.

Apart from simple poles and zeros that account for the overall shape of the open-loop transfer function of a control system, there are a few building blocks common to several of the LIGO length and frequency feedback loops: (i) zeros to compensate cavity poles, (ii) inverted pendulum transfer functions with the gain rising toward higher frequencies to compensate for the $f^{-2}$ fall-off in the response of a suspended optics, (iii) resonant gain sections that add gain in a narrow band to suppress the stack resonances and the vertical pendulum resonances, (iv) narrow-band notch filters that attenuate internal test mass resonances, and (v) steep elliptic low-pass filters that suppress out-of-band noise and that serve as antialiasing filters. Since the differential-mode control system and the parts of the common-mode control system that feed back to the mirror positions are implemented digitally, another set of frequently used building blocks are whitening and dewhitening filters. Their purpose is to shape the signals so that they fit within the limited dynamic range of currently available analog-to-digital and digital-to-analog converters. Using digital filters has the advantage of increased flexibility and the ability to transmit signals over the 4-km distance without degradation. It also makes it relatively easy to switch filters in and out, ramp gains, or even change their shapes during the initial lock acquisition phase when the interferometer transitions from the unlocked to the locked state. ${ }^{30}$

\section{Conclusions}

The coupled-cavity optical topology chosen for the LIGO interferometer permits optimization of its shotnoise-limited sensitivity at expected gravitationalwave frequencies, given constraints of available lasers and optical components. However, sensing and controlling the resulting coupled optical paths and light wavelength to achieve this sensitivity presents a challenge in managing the direct and parametric couplings of diverse environmental influences and instrumental artifacts.

We have presented a design that permits disentanglement and feedback correction of the four relevant lengths and the common laser wavelength for this topology, using signals derived from the antisymmetric output and two other beam samples that have lower signal-to-noise ratios. We have devised a feedback-control system for continuous maintenance of the desired lengths during operation, permitting recovery of the gravitational-wave strain signal at the required sensitivity without contamination by residual laser frequency or intensity noise, without degradation by seismic noise or technical noise from local position sensors, and without pollution by noise from the lower-quality sensing channels. Combined with an active wave-front-based cavity-alignment system, ${ }^{25}$ our design is expected to permit continuous operation at an astrophysical strain sensitivity of $10^{-21} \mathrm{rms}$ in the three LIGO interferometers.

We thank our colleagues on the LIGO project who helped us with many useful suggestions and comments. In particular, we thank Jordan Camp for his early involvement in calculating the effects of light noise sources. This research was supported by National Science Foundation cooperative agreement PHY-9210038 and grant PHY-9870032.

\section{References}

1. A. Abramovici, W. Althouse, J. Camp, J. A. Giaime, A. Gillespie, S. Kawamura, A. Kuhnert, T. Lyons, F. J. Raab, R. L. Savage Jr., D. Shoemaker, L. Sievers, R. Spero, R. Vogt, R. Weiss, S. Whitcomb, and M. Zucker, "Improved sensitivity in a gravitational wave interferometer and implications for LIGO," Phys. Lett. A 218, 157-163 (1996).

2. B. Caron, A. Dominjon, C. Drezen, R. Flaminio, X. Grave, F. Marion, L. Massonnet, C. Mehmel, R. Morand, B. Mours, V. Sannibale, M. Yvert, D. Babusci, S. Bellucci, S. Candusso, G. Giordano, G. Matone, J.-M. Mackowski, L. Pinard, F. Barone, E. Calloni, L. DiFiore, M. Flagiello, F. Garuti, A. Grado, M. Longo, M. Lops, S. Marano, L. Milano, S. Solimeno, V. Brisson, F. Cavalier, M. Davier, P. Hello, P. Heusse, P. Mann, Y. Acker, M. Barsuglia, B. Bhawal, F. Bondu, A. Brillet, H. Heitmann, J.-M. Innocent, L. Latrach, C. N. Man, M. PhamTu, E. Tournier, M. Taubmann, J.-Y. Vinet, C. Boccara, P. Gleyzes, V. Loriette, J.-P. Roger, G. Cagnoli, L. Gammaitoni, J. Kovalik, F. 
Marchesoni, M. Punturo, M. Beccaria, M. Bernardini, E. Bougleux, S. Braccini, C. Bradaschia, G. Cella, A. Ciampa, E. Cuoco, G. Curci, R. DelFabbro, R. DeSalvo, A. DiVirgilio, D. Enard, I. Ferrante, F. Fidecaro, A. Giassi, A. Giazotto, L. Holloway, P. LaPenna, G. Losurdo, S. Mancini, M. Mazzoni, F. Palla, H.-B. Pan, D. Passuello, P. Pelfer, R. Poggiani, R. Stanga, A. Vicere, Z. Zhang, V. Ferrari, E. Majorana, P. Puppo, P. Rapagnani, and F. Ricci, "The VIRGO interferometer for gravitational wave detection," Nucl. Phys. B 54, 167-175 (1997).

3. K. Danzmann, "GEO 600 — a 600-m laser interferometric gravitational wave antenna," in First Edoardo Amaldi Conference on Gravitational Wave Experiments, E. Coccia, G. Pizella, and F. Ronga, eds. (World Scientific, Singapore, 1995), pp. 100111.

4. K. Tsubono, "300-m laser interferometer gravitational wave detector (TAMA300) in Japan," in First Edoardo Amaldi Conference on Gravitational Wave Experiments, E. Coccia, G. Pizella, and F. Ronga, eds. (World Scientific, Singapore, 1995), pp. 112-114.

5. R. Weiss, "Electromagnetically coupled broadband gravitational antennae," MIT Res. Lab. Electron. Q. Prog. Rep. 105, 54-76 (1972).

6. J.-Y. Vinet, B. J. Meers, C. N. Man, and A. Brillet, “Optimization of long-baseline optical interferometers for gravitationalwave detection,” Phys. Rev. D 38, 433-447 (1988).

7. B. J. Meers, "The frequency response of interferometric gravitational wave detectors," Phys. Lett. A 142, 465-470 (1989).

8. R. W. P. Drever, J. L. Hall, F. V. Kowalski, J. Hough, G. M. Ford, A. J. Munley, and H. Ward, "Laser phase and frequency stabilization using an optical resonator," Appl. Phys. B 31, 97-105 (1983).

9. R. L. Savage, P. J. King, and S. U. Seel, "A highly stabilized 10-watt Nd:YAG laser for the laser interferometer gravitational-wave observatory (LIGO)," Laser Phys. 8, 679685 (1998)

10. S. Kawamura, A. Abramovici, and M. E. Zucker, "Improved multistage wide band laser frequency stabilization," Rev. Sci. Instrum. 68, 223-229 (1997).

11. A. Rüdiger, R. Schilling, L. Schnupp, W. Winkler, H. Billing, and K. Maischberger, "A mode selector to suppress fluctuations in laser beam geometry," Opt. Acta 28, 641-658 (1981).

12. K. D. Skeldon, K. A. Strain, A. I. Grant, and J. Hough, "Test of an $18 \mathrm{~m}$ suspended modecleaner cavity," Rev. Sci. Instrum. 67, 2443-2448 (1996).

13. A. Araya, N. Mio, K. Tsubono, K. Suehiro, S. Telada, M. Ohashi, and M.-K. Fujimoto, "Optical mode cleaner with suspended mirrors," Appl. Opt. 36, 1446-1453 (1997).

14. R. W. P. Drever, in Gravitational Radiation, N. Duruell and T. Piran, eds. (North-Holland, Amsterdam, 1983), p. 321.
15. R. W. P. Drever, J. Hough, A. J. Munley, S.-A. Lee, R. Spero, S. E. Whitcomb, H. Ward, G. M. Ford, M. Hereld, N. A. Robertson, I. Kerr, J. R. Pugh, G. P. Newton, B. Meers, E. D. Brook III, and Y. Gürsel, "Gravitational wave detectors using laser interferometers and optical cavities," in Quantum Optics, Experimental Gravity and Measurement Theory, P. Meystre and M. O. Scully, eds. (Plenum, New York, 1983), pp. 503-524.

16. J. Giaime, P. Saha, D. Shoemaker, and L. Sievers, "A passive vibration isolation stack for LIGO: design, modeling, and testing," Rev. Sci. Instrum. 67, 208-214 (1996).

17. A. Gillespie and F. Raab, "Thermal noise in the test mass suspensions of a laser interferometer gravitational-wave detector prototype," Phy. Lett. A 178, 357-363 (1993).

18. A. E. Siegman, Lasers (University Science, Mill Valley, Calif., 1986), Chap. 13, p. 663.

19. L. Schnupp, Max Planck Institute for Quantum Optics, D-85748 Garching, Germany (personal communication, 1986).

20. J. B. Camp, H. Yamamoto, S. E. Whitcomb, and D. E. McClelland, "Analysis of light noise sources in a recycled Michelson interferometer with Fabry-Perot arms," J. Opt. Soc. Am. A 17, 120-128 (1999).

21. P. Melchior, The Tides of the Planet Earth (Pergamon, Oxford, UK, 1978), p. 279.

22. M. W. Regher, F. J. Raab, and S. E. Whitcomb, "Demonstration of a power-recycled Michelson interferometer with FabryPerot arms by frontal modulation," Opt. Lett. 20, 1507-1509 (1995).

23. M. W. Regher, "Signal extraction and control for an interferometric gravitational wave detector," Ph.D. dissertation (California Institute of Technology, Pasadena, Calif., 1995).

24. R. Flaminio and H. Heitmann, "Longitudinal control of an interferometer for the detection of gravitational waves," Phys. Lett. A 214, 112-122 (1996).

25. P. Fritschel, G. González, N. Mavalvala, D. Shoemaker, D. Sigg, and M. Zucker, "Alignment of a long-baseline gravitational wave interferometer," Appl. Opt. 37, 6734-6747 (1998).

26. J.-Y. Vinet, P. Hello, C. N. Man, and A. Brillet, "A highaccuracy method for the simulation of non-ideal optical cavities," J. Phys. I 2, 1287-1303 (1992).

27. G. F. Franklin, J. D. Powell, and A. Emami-Naeini, Feedback Control of Dynamic Systems, 3rd ed. (Addison-Wesley, Reading, Mass., 1994), p. 356.

28. T. Lay and T. C. Wallace, Modern Global Seimology (Academic, San Diego, Calif., 1995), p. 179.

29. G. I. Gonzalez and P. R. Saulson, "Brownian motion of a mass suspended by an anelastic wire," J. Acoust. Soc. Am. 96, 207212 (1994).

30. D. Redding and L. Sievers, Jet Propulsion Laboratory, 4800 Oak Grove Drive, Pasadena, Calif. 91109 (personal communication, 1997). 